\title{
Two Nonlinear Optimization Methods for Black Box Identification Compared
}

\author{
Anne Van Mulders* Johan Schoukens ** Marnix Volckaert *** \\ Moritz Diehl ${ }^{* * * *}$ \\ * Vrije Universiteit Brussel, Department ELEC, Pleinlaan 2, B1050 \\ Brussels, BELGIUM (e-mail: anne.vanmulders@vub.ac.be) \\ ** Vrije Universiteit Brussel, Department ELEC, Pleinlaan 2, B1050 \\ Brussels, BELGIUM (e-mail: johan.schoukens@vub.ac.be) \\ *** K.U.Leuven, Department MECH, Celestijnenlaan 300b, B3001 \\ Leuven, BELGIUM (e-mail: Marnix.Volckaert@mech.kuleuven.be) \\ **** K.U.Leuven, Department ESAT, Kasteelpark Arenberg 10, B3001 \\ Leuven, BELGIUM (e-mail: Moritz.Diehl@esat.kuleuven.be)
}

\begin{abstract}
In this paper, two nonlinear optimization methods for the identification of nonlinear systems are compared. Both methods estimate all the parameters of a polynomial nonlinear state-space model by means of a nonlinear least-squares optimization. While the first method does not estimate the states explicitly, the second estimates both states and parameters adding an extra constraint equation. Both methods are introduced and their similarities and differences are discussed utilizing simulation and experimental data. The unconstrained method appears to be faster and more memory efficient, while the constrained method is robust towards instabilities.
\end{abstract}

Keywords: Identification algorithms; parameter estimation; nonlinear systems; nonlinear models; state-space models; constraints.

\section{INTRODUCTION}

Most real-life systems are to some extent nonlinear. For such systems, nonlinear modelling can improve the identification results, i.e. reduce the residual error. There exist several types of nonlinear models, among them black box models such as Volterra systems, block structured models, neural networks and fuzzy models. For more information, we refer to Giannakis et al. [2001].

In this paper, the central model structure is a conventional state-space model extended with polynomial nonlinear terms. It is called a Polynomial Nonlinear State-space Model (PNLSS), see Paduart [2008].

The goal is to estimate the parameters of the nonlinear model given the exact input and the noisy output measurements. The states are assumed to be unknown and in order to solve the problem we will minimize the least square error between the measured and modelled output. The performances (least square errors) of two different optimization methods for estimating the parameters of the PNLSS model structure will be compared.

The structure of the paper is the following: in Section 2 , we present the model structure. In Section 3, we explain briefly the generation of initial estimates for the parameters to be estimated. In Section 4, the two nonlinear optimization methods are discussed: the first one (A) less detailed than the second one (B) because the former has already been described extensively in Paduart [2008]. In Sections 5 and 6 , the simulation and experimental results are discussed. The last section recapitulates the main conclusions of this paper.

\section{MODEL STRUCTURE}

\subsection{Class of discrete time systems considered}

As it is known very well, state-space models are particularly well suited for multiple-input multiple-output (MIMO) systems. Let $n_{u}$ and $n_{y}$ represent resp. the number of input and output signals. In general, a discrete time nonlinear state-space model can be formulated as:

$$
\begin{array}{ll}
x(t+1) & =f(x(t), u(t)) \\
y(t) & =h(x(t), u(t))
\end{array}
$$

Herein, $t$ is the discrete time instant. The upper equation is called the state equation and describes the evolution of the states. The lower equation is called the output equation and describes the output as function of the states and inputs.

In our case, we assume that the exact description of the nonlinear system is of the form:

$$
\begin{array}{ll}
x(t+1) & =A x(t)+B u(t)+E \zeta(x(t), u(t)) \\
y(t) & =C x(t)+D u(t)+F \eta(x(t), u(t))
\end{array}
$$

The vectors $\zeta \in \mathbb{R}^{n_{\zeta}}$ and $\eta \in \mathbb{R}^{n_{\eta}}$ contain monomials in $x(t)$ and $u(t)$; the matrices $E \in \mathbb{R}^{n_{a} \times n_{\zeta}}$ and $F \in \mathbb{R}^{n_{y} \times n_{\eta}}$ contain the coefficients associated with those monomials. The above mentioned model is called a polynomial nonlinear state-space model (PNLSS). It consists of a classical linear state-space model with nonlinear terms $E \zeta$ and $F \eta$. The coefficients of the linear terms in $x(t)$ (the states) and $u(t)$ (the inputs) are given by the coefficient matrices $A \in \mathbb{R}^{n_{a} \times n_{a}}$ and $B \in \mathbb{R}^{n_{a} \times n_{u}}$ in the state equation, and $C \in \mathbb{R}^{n_{y} \times n_{a}}$ and $D \in \mathbb{R}^{n_{y} \times n_{u}}$ in the output equation. $n_{a}$ is the number of states (model order) at one time instant, 
$n_{\eta}$ and $n_{\zeta}$ are the number of monomials in resp. $\eta$ and $\zeta$. The monomials can be any chosen set of combinations of $x_{1}^{\alpha_{1}} x_{2}^{\alpha_{2}} \ldots x_{n}^{\alpha_{n}} u_{1}^{\beta_{1}} u_{2}^{\beta_{2}} \ldots u_{n_{u}}^{\beta_{n_{u}}}$ with $\alpha_{1}, \ldots, \alpha_{n}, \beta_{1}, \ldots, \beta_{n_{u}} \in$ $\mathbb{N}$ and $\sum_{j} \alpha_{j}+\sum_{i} \beta_{i} \leq d$. Herein, $d \in \mathbb{N}$ is called nonlinear degree and has to be chosen by the user. The $n$ state equations and $n_{y}$ output equation of a linear state-space system are extended by adding a polynomial to every equation. The major advantage of the PNLSS model is its capability of describing a very large class of nonlinear systems, such as bilinear models, affine models, nonlinear models with only nonlinearities in the states, nonlinear models with only nonlinearities in the input and certain block-structured nonlinear models (Wiener, Hammerstein, Wiener-Hammerstein and nonlinear feedback), see Paduart [2008]. Consequently, it can be stated that the PNLSS model structure is a generic all-purpose black-box model structure.

\subsection{Parameterization}

Despite the ease with which the model structure can handle MIMO systems, we will restrict ourselves without loss of generality to single-input single-output (SISO) systems $\left(n_{u}=n_{y}=1\right)$ in order to focus on the main topic of this paper.

Define $\theta \in \mathbb{R}^{n_{\theta}}$ as a vector containing all the model parameters:

$$
\theta^{T}=\left[\operatorname{vec}(A)^{T} B^{T} C D \operatorname{vec}(E)^{T} F\right]
$$

with vec an operator that stacks the columns of a matrix onto each other.

\subsection{Stochastic framework}

The input is assumed to be known exactly (without noise). If the model is capable of describing the system, the output measurements $y_{m}$ are related to the system output $y\left(t, \theta_{0}\right)$ :

$$
y_{m}(t)=y\left(t, \theta_{0}\right)+v(t)
$$

with $\theta_{0}$ the true parameter values and $v(t)$ the output measurement noise, which is here for simplicity assumed to be white, zero mean and with finite variance.

\section{INITIAL ESTIMATES}

In both cases (see Paduart [2008]), the initial estimates for the linear parameters $(A, B, C, D)$ are found by a twostep procedure.

First, the Best Linear Approximation (BLA) (see Pintelon et al. [2001]) of the system is estimated.

$$
\hat{G}_{B L A}(k)=\frac{\hat{S}_{Y U}(k)}{\hat{S}_{U U}(k)}
$$

with $\hat{G}_{B L A}$ the estimated frequency response function, $k$ the frequency line, $\hat{S}_{Y U}$ the estimated cross-power spectrum between output and input and with $\hat{S}_{U U}$ the estimated auto-power spectrum of the input. The BLA minimizes the output error in least squares sense. Also the variance $\hat{\sigma}_{G_{B L A}}^{2}(k)$ can be estimated to enhance the second step by using a weighted least-squares method.

Second, this nonparametric model is converted into a linear parametric state-space model using the Frequency Domain Subspace identification method (McKelvey et al.
[1996] and Pintelon [2002].

Using a frequency domain approach is equivalent to using a time domain approach, but it has two advantages: bounded initial estimates can also be obtained for unstable systems and weighting is made easier.

The nonlinear parameter matrices $(E$ and $F)$ are initialized to zero.

\section{NONLINEAR OPTIMIZATION}

\subsection{Cost function}

We define the measured output as

$$
y_{m}^{T}=\left[y_{m}(0) \cdots y_{m}(N-1)\right]
$$

and the modelled output as

$$
y(\theta)^{T}=[y(0, \theta) \cdots y(N-1, \theta)]
$$

$N$ is the number of time samples. Both methods intend to minimize the same least squares cost function:

$$
V(\theta)=\epsilon^{T}(\theta) \epsilon(\theta)
$$

with $\epsilon(\theta)=y(\theta)-y_{m}$

Methods A and B differ in two respects: the choice of the free variables and the lack or presence of constraints. In method A, the free variables are only the model parameters, in method B, both the model parameters and the states are free variables.

\subsection{Method A: (Unconstrained) Levenberg-Marquardt}

The cost function (8) of the least squares problem is nonlinear in the parameters. Using a nonlinear optimization method, such as the Levenberg-Marquardt method, a (local) minimum is found in an iterative way:

$$
\left(J^{T} J+\lambda_{L M}^{2} I_{n_{\theta}, n_{\theta}}\right) \delta \theta=-J^{T} \epsilon
$$

with $\lambda_{L M}$ the Levenberg-Marquardt factor and $J$ the Jacobian matrix:

$$
J=\frac{\partial \epsilon}{\partial \theta}
$$

The parameters are updated by adding $\delta \theta$ to the previous value of $\theta . \delta \theta$ is calculated by a singular value decomposition (SVD) of the Jacobian matrix. See Paduart [2008].

\subsection{Method B: Constrained Levenberg-Marquardt (CLM)}

The second method is inspired by the so called "simultaneous approach" to parameter estimation in differential equations (see Bock et al. [1992], Bock [1983, 1987]), which we shall call the "constrained LevenbergMarquardt" method.

The optimization variables are the states (at all time instants) and the parameters of the chosen model:

$$
\vartheta^{T}=\left[\begin{array}{lll}
x^{T}(0) & \cdots & x^{T}(N-1) \theta^{T}
\end{array}\right]
$$

In this case, (7) and (8) should be interpreted as functions of $\vartheta$ instead of $\theta$.

In the CLM method, cost function (8) is minimized subject to the constraint function $F(\vartheta)=0$, with:

$$
F(\vartheta)=\left(\begin{array}{c}
f(x(0), u(0))-x(1) \\
f(x(1), u(1))-x(2) \\
\vdots \\
f(x(N-1), u(N-1))-x(0)
\end{array}\right)
$$


This constraint function is built up by the differences between the modelled states (see state equation in (1)) and the states of the previous iteration. The last constraint equation imposes periodicity. For aperiodic signals, this equation should be deleted.

The initial estimates of the parameter values $\theta$ are determined as in Section 3. For periodic signals, the initial values of the states can be calculated in the time domain by using the state equation of $(2)$, with $u(t)$ the known input, $A, B$ (and $E=0)$ parameters in $\theta$. Transient effects can be eliminated by simply simulating several periods (starting from $x(0)=0$ ) and retaining only the last period. An alternative in the time domain is to estimate the initial state $x(0)$ by adding an artificial input to the model (see Paduart [2008]) and then proceeding as described above to obtain the states at the other time instants. Another option is to calculate the states in the frequency domain (z-domain) via:

$$
X(z)=(z I-A)^{-1} B U(z)
$$

with $X$ and $U$ the discrete Fourier transforms of the time domain signals $x$ and $u$. This provides bounded initial estimates, even in case of unstable systems (within a stabilizing feedback).

For aperiodic signals, the time-domain implementation that starts from an estimate of $x(0)$ can be used for stable systems. However, this method does not work in the unstable case. We choose to use the frequency domain approach for both periodic and aperiodic signals in this paper. For periodic signals, the resulting estimates are exact but for aperiodic signals, we assume that the transient errors will be corrected in the iterations of the constrained minimization. Anyhow, the initial parameter values of $\theta$ are incorrect so we assume that the extra mistakes made by transient errors will not be important.

The (constrained) minimization is achieved by iteratively updating the variable vector $\vartheta$. In the minimization process, a new value of $\vartheta$ is calculated by adding $\delta \vartheta$ to the current value. If we would use the constrained Gauss-Newton method (see Bock [1983]), $\delta \vartheta$ would be the solution of:

$$
\underbrace{\left(\begin{array}{cc}
J_{1}^{T} J_{1} & -J_{2}^{T} \\
J_{2} & 0_{N_{s t}, N_{s t}}
\end{array}\right)}_{K K T}\left(\begin{array}{c}
\delta \vartheta \\
\lambda
\end{array}\right)=\left(\begin{array}{c}
-J_{1}^{T} \epsilon \\
-F
\end{array}\right)
$$

with KKT the Karush-Kuhn-Tucker matrix (see Fletcher [1991]) and $J_{1} \in \mathbb{R}^{N \times n_{\vartheta}}$ and $J_{2} \in \mathbb{R}^{N_{s t} \times n_{\vartheta}}$ the Jacobians of the residual vector $\epsilon$ and constraint function:

$$
J_{1}=\frac{\partial \epsilon}{\partial \vartheta}, \quad J_{2}=\frac{\partial F}{\partial \vartheta}
$$

$N_{s t}$ is the total number of states (at all time instants): $N_{s t}=N n_{a}$. The Lagrange multipliers in vector $\lambda \in$ $\mathbb{R}^{N_{s t} \times 1}$ are a byproduct of the calculations and are a measure for the sensitivity of the objective to changes in each constraint.

It might become time-consuming to invert the KKT matrix because it grows (in both dimensions) with the number of data points $N$. On the other hand, the matrix is very sparse, which allows for the use of more efficient direct inversion techniques available in software packages such as Matlab $^{\mathrm{TM}}$. Also, the specific structure of the KKT system can be exploited by tailored KKT solvers, as e.g. proposed by Bock [1987]. Fig. 1 depicts the nonzero elements of

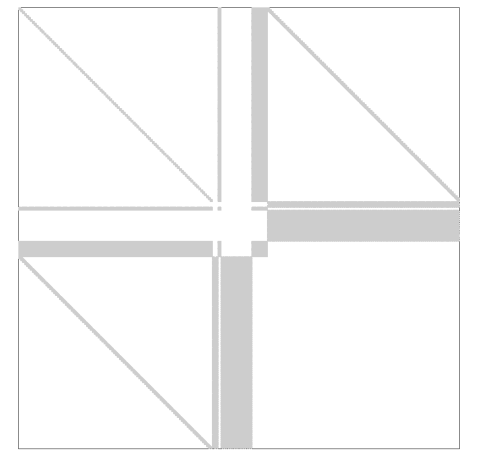

Fig. 1. Position of the nonzero elements (grey dots) of the KKT matrix.

the KKT matrix for 100 data points, model order 2 and nonlinearities of degrees 2 and 3 .

Unfortunately, the indeterminations of the state-space model (due to similarity transforms) cause the KKT matrix in (14) to be singular. In a normal situation, one would immediately consider using the pseudo-inverse, but even for sparse matrices, singular value decompositions require a huge amount of computation time. However, adding a Levenberg-Marquardt parameter to the diagonal of $J_{1}^{T} J_{1}$ can render the KKT matrix nonsingular. By choosing $\lambda_{L M}$ sufficiently large, the conditioning of the KKT matrix is improved without adding too many nonzero elements. This does not significantly increase the inversion time.

$$
\left(\begin{array}{cc}
J_{1}^{T} J_{1}+\lambda_{L M}^{2} I_{n_{\vartheta}, n_{\vartheta}} & -J_{2}^{T} \\
J_{2} & 0_{N_{s t}, N_{s t}}
\end{array}\right)\left(\begin{array}{c}
\delta \vartheta \\
\lambda
\end{array}\right)=\left(\begin{array}{c}
-J_{1}^{T} \epsilon \\
-F
\end{array}\right)
$$

This is not equivalent to a Levenberg-Marquardt method for unconstrained optimization, where a larger $\lambda_{L M}$ tends the algorithm to follow the gradient descent direction (with a larger convergence region than the Gauss-Newton method). In constrained optimization, convergence is no longer guaranteed by just adding a sufficiently high $\lambda_{L M}$ (see Fletcher [1991]). We encountered convergence problems, but could solve them by means of a line search.

\section{SIMULATIONS}

The first method was already implemented (see Paduart [2008]). Some simulations are nevertheless carried out with method A to pinpoint possible advantages or disadvantages between methods A and B. For method B, the simulations are especially a check to verify whether convergence is obtained (in a noiseless and in a noisy case).

The idea is to start with a known PNLSS model structure so that - for the right model settings - the cost function should converge to zero (or computer precision) in the noiseless case and to noise level in the noisy case. The constraint function should also converge to computer precision, even in the noisy case. The results of methods $\mathrm{A}$ and $\mathrm{B}$ are in general comparable, but nevertheless, some cases could be found where method A could not converge while method B had no difficulties. One such a result is shown in Fig. 2, in which the convergence curves of methods $\mathrm{A}$ and $\mathrm{B}$ are plotted (cost versus iteration number) in a noiseless case. The selected model structure is of order 2 and has nonlinear degrees 2 and 3 . The cost is based on the data 


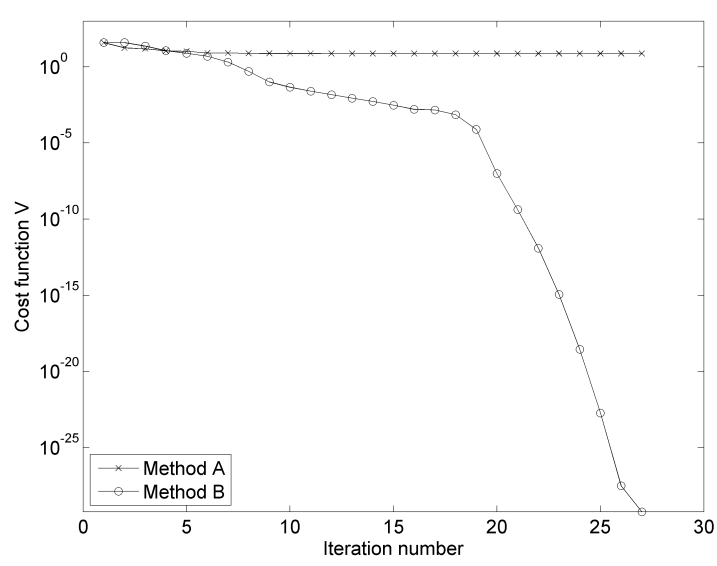

Fig. 2. Convergence curve: method A (crosses) and method B (circles).

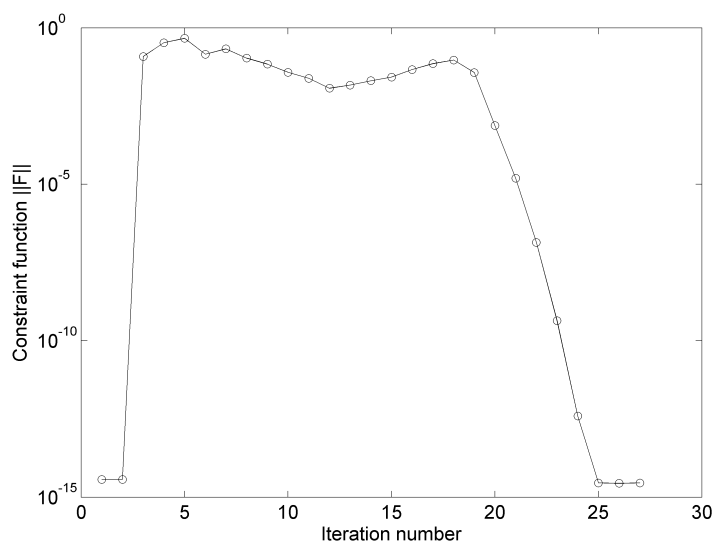

Fig. 3. Constraint versus iteration number: method B.

used in the estimation; if validation (or test) data would be used, the cost function of method B would not necessarily decrease monotonically, but would still converge (in this example). The evolution of the constraint function of method B is depicted in Fig. 3.

The difficulty to identify the chosen model can be explained by its 'nearly unstable' nature: the poles of the underlying linear system lie close to the unit circle (at radius 0.967 ) and the estimated models are often unstable.

We now give an example of an unstable linear system that can be identified with method B.

An unstable linear system is placed in a stabilizing feedback loop. Since the system is linear, the subspace method already provided an estimate of its state-space parameters. When we start from perturbations of these parameters, method B manages to converge, while method A cannot handle the unstable system. The curves of method B are similar to the circles in Fig. 2 and Fig. 3.

The problem with method $\mathrm{A}$ is that it calculates the output by means of the entire state-space model (2). If the system is unstable, the states and consequently the output (and output error) will grow very (even infinitely) large. In method $\mathrm{B}$, the states are considered to be model parameters, and the output (and output error) are calculated by means of the output equation only. If the initial state estimates are bounded, so will be the output. Said in other words, method A suffers from the ill-posedness of the simulation problem for an unstable system, while method B does not.

\section{EXPERIMENTAL RESULTS}

\subsection{The Device Under Test}

The DUT is an electronic circuit with a Wiener-Hammerstein structure. A Wiener-Hammerstein system is a nonlinear system consisting of two linear dynamic systems and a static nonlinearity in between. The DUT is currently used in a benchmark (Schoukens et al. [2009]).

A measured set of input and output data was split up in estimation data (100000 samples) and test (or validation) data (88000 samples). The input was a filtered Gaussian excitation signal with cut-off frequency $10 \mathrm{kHz}$ and sample frequency $51.2 \mathrm{kHz}$. The root mean square (RMS) value of the input signal is $0.66 \mathrm{~V}$.

\subsection{Model Selection}

For the model selection, the 100000 data points of the estimation set are split up in two blocks. The first block for the estimation of several model structures (combinations of model order and nonlinear terms), the other block for the selection of the best model. The model structure with the smallest RMS error $\left(e_{R M S}\right)$ on this second block is chosen.

\subsection{Linear Model}

A 6th order linear state-space model is calculated as explained in Section 3. This linear model will be suitable as starting-point for both the unconstrained and constrained optimization methods. The linear model is validated on the test data and the measured output of the system is plotted in Fig. 4 (black line) together with the simulation error (grey line). The RMS value of the simulation error $\left(e_{R M S t}\right)$ is about $23 \%$ of the RMS output level that measures $0.24 \mathrm{~V}$. This linear model contains 49 parameters, but only 13 parameters are necessary to describe the input-output behaviour due to the similarity transform (characterized by a transformation matrix with $n_{a}^{2}=36$ elements). Other model orders were tried out, but none of them could significantly improve this poor result. It is however wrong to conclude that all nonlinear models based on this 6 th order linear model perform better than higher order models. Table 1 shows the RMS value of the simulation (test) error $\left(e_{R M S t}\right)$, its mean value $\left(\mu_{t}\right)$, its standard deviation $\left(s_{t}\right)$ and the RMS value of the estimation error $\left(e_{R M S e}\right)$. They are defined as in the benchmark Schoukens et al. [2009], except that the $e_{R M S e}$ is computed on the $N$ data points that were used to obtain this linear model (minus 1000 points to eliminate transient effects).

\subsection{Nonlinear Model}

When the nonlinear model is an extension of a linear model of order 6 , the nonlinear terms of the PNLSS model consist of combinations between the 6 states $x_{1}(t), \ldots, x_{6}(t)$ and 

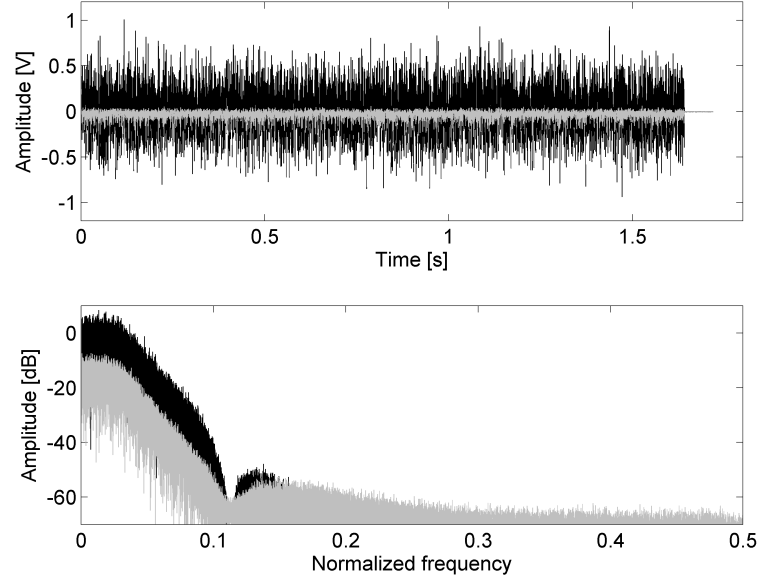

Fig. 4. Validation result for the 6th order linear model: modelled output (black) and simulation error (grey); time (top) and frequency domain (bottom).

the input $u(t)$ in both the state and output equation of (2). Every monomial (such as $x_{1} x_{3}^{2} u$ ) is weighted by a coefficient of the parameter matrices $E$ and $F$ and the sums of these terms result in polynomials.

Method A: Levenberg-Marquardt This method was already tried out on the same DUT, but with different measurement data: several periods of a multisine and lower excitation amplitudes. This is described in Paduart [2008]. Since the purpose of this paper is to compare methods A and $\mathrm{B}$, the identification is repeated. The chosen model order and nonlinear degrees are different. The order of the linear model is kept at 6 and the nonlinear degree is - if necessary - set higher than in the reference. This because in method $\mathrm{B}$, the cost (in terms of calculation time and memory) of adding nonlinear degrees is much lower than the cost of augmenting the order. Besides, increasing the nonlinear degree had a higher impact than increasing the linear order in the examples in Paduart [2008].

The best model structure (of the examined ones) appears to be a model with nonlinear degree $d=3$ in the state and output equation. This model contains 833 parameters, or 797 independent parameters. The validation of this nonlinear model is plotted in Fig. 5 (black line) together with the simulation error (grey line). The top figure is a time domain plot, the bottom figure is the frequency domain result. The $e_{R M S t}$ is reduced considerably: it is about $0.2 \%$ of the RMS output level and $0.8 \%$ of the linear $e_{R M S t}$. The results are shown in Table 1, with the $e_{R M S e}$ based on 95000 estimation points.

Method B: Constrained Levenberg-Marquardt For estimations with a large amount of data points and a high order of the underlying linear system, the dimension of the KKT matrix will soon be very large. Even if sparse techniques from Matlab are applied, the calculation time grows approximately quadratically with the amount of data points and the computer could even run out of memory. Thus, we restrict ourselves to a shorter dataset than the one we used in method A.

Since the signals are aperiodic, the optional periodicity constraint in (12) was deleted.
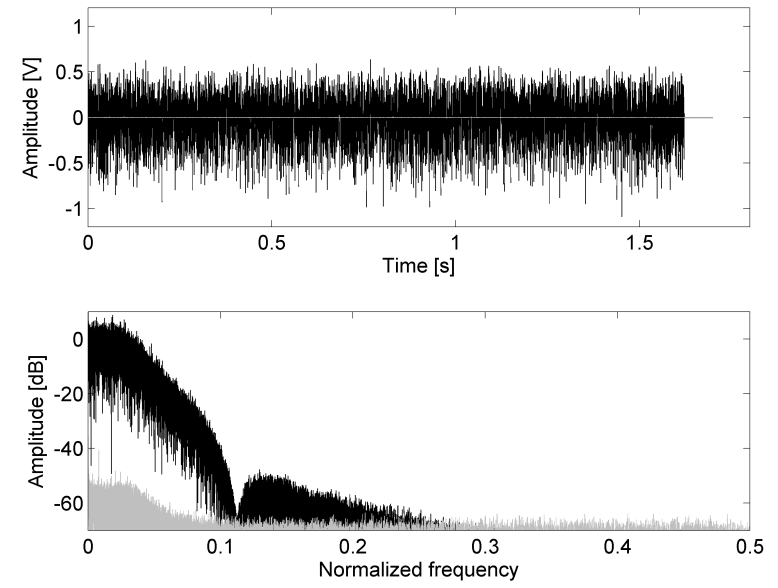

Fig. 5. Validation result for the nonlinear model of method A: measured output (black) and model simulation error (grey).
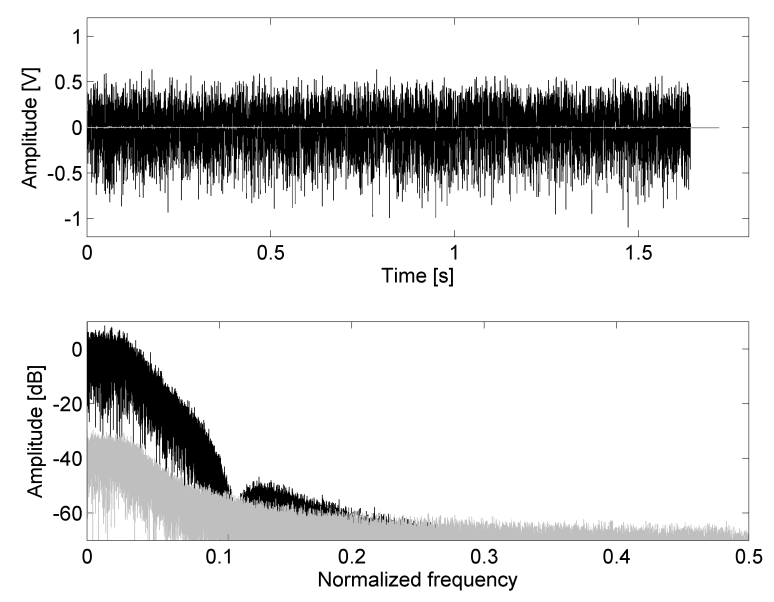

Fig. 6. Validation result for the nonlinear model of method B: measured output (black) and model simulation error (grey).

With the same settings as in method A (nonlinear degree $d=3$ for the state and output equation), but with only 6000 instead of 100000 data points, the RMSE is about $1.1 \%$ of the RMS output level and still a significant reduction compared to the linear error. The results are shown in Fig. 6 and Table 1 (6000 points used to compute the $\left.e_{R M S e}\right)$.

This result was obtained by using an estimated model obtained on another 6000 points as an initial estimate. Improvements of the result are possible by choosing other data points (out of the 100000).

\subsection{Comparison}

To be able to compare the two methods, method $\mathrm{A}$ is run with the 6000 data points used in method B. The results are plotted in Fig. 7 and shown in Table 1 (6000 points used to compute the $\left.e_{R M S e}\right)$. The results are very similar to the results of method $\mathrm{B}$. 

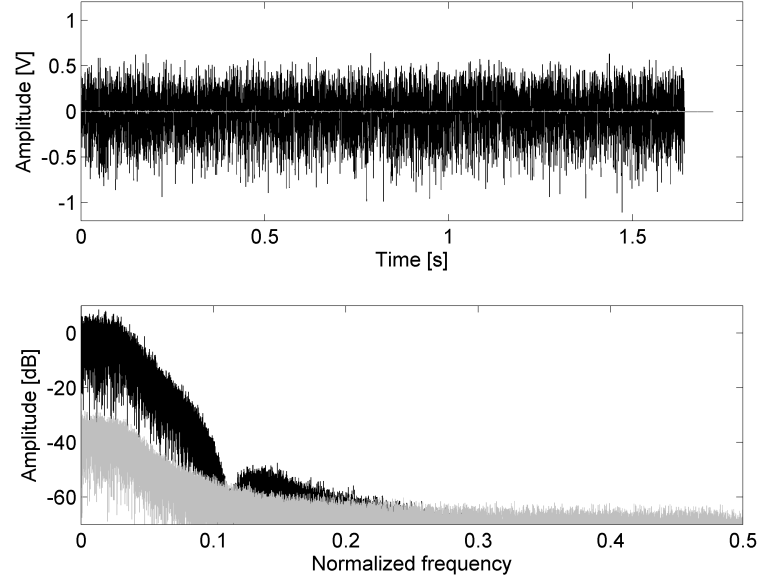

Fig. 7. Validation result for the nonlinear model of method A (6000 estimation points): measured output (black) and model simulation error (grey).

\begin{tabular}{|c|c|c|c|c|c|}
\hline Model & $N$ & $\begin{array}{c}e_{R M S t} \\
{[m V]}\end{array}$ & $\begin{array}{c}\mu_{t} \\
{[m V]}\end{array}$ & $\begin{array}{c}s_{t} \\
{[m V]}\end{array}$ & $\begin{array}{c}e_{R M S e} \\
{[m V]}\end{array}$ \\
\hline Linear & 95000 & 56.2 & -36.0 & 43.2 & 57.3 \\
NL, A & 95000 & 0.443 & -0.0523 & 0.440 & 0.323 \\
NL, A & 6000 & 3.11 & -0.249 & 3.10 & 0.268 \\
NL, B & 6000 & 2.62 & 0.00441 & 2.62 & 0.630 \\
\hline
\end{tabular}

Table 1. Properties of the residuals for 6 th order models. Subscripts $t$ and $e$ refer resp. to the test and estimation sets.

The convergence curves on the test data (not shown in this paper) behave differently depending on the number of data points used. The curves of method A with 95000 estimation points decrease nicely, while the curves of the two methods on 6000 estimation points show a very irregular behaviour, not even close to a monotonic decrease and some of the estimated models are unstable on the test set. The cause may be that 6000 estimation points are insufficient for a reliable estimation with the current (rather high) amount of parameters. The problem does not occur with more parameter-parsimonious models such as state affine models: the $e_{R M S t}(5.2 \mathrm{mV})$ is slightly increased compared to the full third degree model.

Anyhow, the limited number of data points of method B is one of its weaknesses. It might be overcome with structure exploiting algorithms (see Bock et al. [1992]).

On the other hand, Section 5 showed an advantage of method B: its robustness towards instabilities.

\section{CONCLUSION}

The results of constrained and unconstrained optimization with the same settings were very comparable. Nevertheless, they differ in some respects:

- When using off-the-shelf sparse direct solvers, the constrained optimization cannot handle very large amounts of data points, because of an increased calculation time and memory limitations.

- The constrained optimization showed to be robust in case of nearly unstable nonlinear systems with stable underlying linear part, while unconstrained optimiza- tion sometimes failed. The constrained optimization was able to identify a linear unstable system within a control loop, unlike the unconstrained optimization. We expect the constrained optimization to be robust in case of unstable nonlinear systems within a control loop.

Further research will focus on combining the positive aspects of both methods by gradually moving from a constrained optimization with few data points and all constraint equations, towards the unconstrained method with many data points. That way, the optimization process can cross unstable regions and the variance of the results will be smaller because in the end, the entire data set can be used.

\section{ACKNOWLEDGEMENTS}

This research was supported by the Methusalem grant of the Flemish Government (METH-1), the Belgian Program on Interuniversity Poles of Attraction initiated by the Belgian State, Prime Minister's Office, Science Policy programming (IUAP VI/4 - Dysco), the research council of the Vrije Universiteit Brussel (OZR), the Fund for Scientific Research (FWO - Vlaanderen), IUAP P6/04 (DYSCO), KUL EF/05/006 (OPTEC), IOF-SCORES4CHEM, FWO G.0320.08 (convex MPC), G.0558.08 (Robust MHE), ICCoS, EU FP7 223854 (HDMPC), Helmholtz viCERP and Comet ACCM.

\section{REFERENCES}

H. G. Bock, T. Lohmann and J. P. Schlöder. Numerical methods for parameter estimation and optimal experimental design in chemical reaction systems. Industrial and Engineering Chemistry Research, volume 31, issue 3, pages 54-57, 1992.

H. G. Bock. Numerical treatment of inverse problems in differential and integral equations. Birkhäuser, 1983.

H. G. Bock, T. Lohmann and J. P. Schlöder. Randwertproblemmethoden zur Parameteridentifizierung in Systemen nichtlinearer Differentialgleichungen. Universität Bonn, volume 183, 1987.

R. Fletcher. Practical methods of optimization. John Wiley and Sons, 1991.

G. B. Giannakis and E. Serpedin. A bibliography on nonlinear system identification. IEEE Trans. Signal Process., volume 81, issue 3, pages 533-580, 2001.

T. McKelvey, H. Akay and L. Ljung. Subspace-based multivariable system identification from frequency response data. IEEE Trans. Autom. Control, volume 41, issue 7, pages 960-979, 1996.

J. Paduart. Identification of nonlinear systems using polynomial nonlinear state space models. $\mathrm{PhD}$-thesis, Vrije Universiteit Brussel (VUB), 2008.

R. Pintelon. Frequency-domain subspace system identification using non-parametric noise models. Automatica, volume 38, issue 8, pages 1295-1311, 2002.

R. Pintelon and J. Schoukens. System Identification: a frequency domain approach. IEEE Press, New Jersey, 2001.

J. Schoukens, J. Suykens and L. Ljung. WienerHammerstein benchmark. SYSID 2009, 2009. 